\title{
Application of MPC to Spacecraft Attitude Maneuver using RCS and/or RW*
}

\author{
Takeya ASAKAWA** and Takashi KIDA** \\ ** University of Electro-Communications \\ 1-5-1 Chofugaoka, Chofu, Tokyo 182-8585 Japan \\ E-mail: takeya-a@ctr.mce.uec.ac.jp
}

\begin{abstract}
This paper applies model predictive control (MPC) to multi-input spacecraft attitude maneuver problems using reaction control systems (RCS) and reaction wheels (RW). For this purpose, we consider linear quadratic cost functions under the input constraints of nonlinear on-off control inputs for RCS and linear continuous inputs with saturation for RW. These are optimized as mixed integer quadratic programming (MIQP) problems. We further analyze the closed-loop stability in the input-to-state stability framework and derive a stability condition described by a linear matrix inequality (LMI). Some numerical studies are performed and the effectiveness of the proposed model predictive control is shown.
\end{abstract}

Key words : Spacecraft Attitude Maneuver, Reaction Control Systems, Reaction Wheels, Model Predictive Control, Input-to-State Stability, Mixed Integer Quadratic Programming

\section{Notation}

$\mathbb{R}, \mathbb{R}^{n}:$ Real numbers and real $n$-vectors.

$\mathbb{R}^{n \times n}:$ Real $n \times n$ matrix.

$\mathbb{R}_{\geq 0}:$ Non-negative real numbers.

$\mathbb{Z}, \mathbb{Z}^{n}:$ Integer numbers and $n$-vectors.

$\mathbb{Z}_{+}$: Positive integer numbers.

$\mathbb{R}^{n} \mapsto \mathbb{R}: \mathbb{R}^{n}$ maps to $\mathbb{R}$

$M>0$ : Matrix $M$ is positive definite.

$M \geq 0$ : Matrix $M$ is positive semi-definite.

$M>N: M-N>0$ for matrices $M$ and $N$.

\section{Introduction}

Spacecraft attitude maneuvers are frequently performed during operational phases such as initial attitude acquisition or antenna calibration ${ }^{(1)}$. We also find spacecraft missions that require high-rate large-angle changing maneuvers to be executed with high accuracy in the fields of Earth and astronomical observation ${ }^{(2),(3)}$. In order to achieve large angular maneuvers of heavy spacecraft, reaction control systems (RCS), with their ability to produce large moments of force, are the standard actuators. While RCS can only generate nonlinear on-off inputs, they are the principal means of achieving open-loop attitude rotations ${ }^{(4)}$. The use of linear feedback control is made possible with the aid of pulse modulation technology ${ }^{(1)}$, but in this case the closed-loop stability and the control performance expected when designing linear control laws can no longer be assured. On the other hand, reaction wheels (RW) are widely used for precise attitude feedback control. Although a linear feedback control law can be successfully achieved by RW, they are not appropriate for large-angle maneuvers since the maximum torque is limited, and it is not easy to ensure closed-loop stability and control performance when control inputs are saturated.
$M \geq N: M-N \geq 0$ for matrices $M$ and $N$. $x>y: x_{i}>y_{i}$ for all elements of vectors $x$ $I_{n}: n \times n$ identity matrix.

$|x|$ : Euclidean norm of vector $x$.

$\|M\|$ : Induced norm of matrix $M$.

$\lambda_{\max }(M)$ : Maximum eigenvalue of $M$.
*Received 25 Feb., 2013 (No. 13-0097)

[DOI: 10.1299/spacee.6.1]

Copyright (c) 2013 by JSME 
In view of these issues, this paper considers applying model predictive control (MPC) to attitude maneuvers using RCS and RW singly and in combination. The advantage of applying MPC lies in the fact that optimal control is possible by explicitly treating the nonlinear input constraints caused by RW saturation and the on-off property of RCS. By utilizing this feature, the most effective attitude maneuver is expected to be achieved. Although there are other research works on the application to spacecraft attitude control of explicit $\mathrm{MPC}^{(5)}$ and nonlinear MPC ${ }^{(6),(7)}$ considering input non-linearity, the former requires large memory capacity and therefore the study is limited to a single-input case, and the latter has difficulty in on-line optimization.

In this paper, we first formulate the problem using tractable linear quadratic cost functions under the input constraints of nonlinear on-off control inputs and linear continuous inputs with saturation. The cost functions are optimized as mixed integer quadratic programming (MIQP) problems which are numerically solvable using the branch and bound method. We then investigate the closed-loop stability of MPC for attitude maneuvers which is tightly related to the terminal cost. Regarding stability, there already exists a stability condition using a solution of the discrete algebraic Riccati equation (DARE) for linear continuous inputs ${ }^{(8),(9)}$, which is the case for RW control, but stability for the case of on-off inputs such as RCS control has not yet been investigated. For this problem, we newly propose to construct a terminal cost matrix based on a solution of a linear matrix inequality (LMI). This gives an input-to-state stability (ISS) condition ${ }^{(10)-(12)}$ for on-off RCS inputs. It is then shown to be a unified condition that includes asymptotic stability (AS) for linear continuous inputs by RW, and gives greater flexibility in designing terminal cost than the DARE. Finally, some numerical study results are shown to confirm the effectiveness of the proposed MPC by applying it to a spacecraft multi-input attitude maneuver using RW and RCS singly and in combination.

\section{Problem Description}

The MPC for a simple rigid spacecraft attitude maneuvering problem is considered. The dynamical equation linearized at the equilibrium point $(\theta, \dot{\theta})=0$ is

$$
J \ddot{\theta}=u_{\{r c s, \max \}} u_{r c s}+u_{r w}
$$

where $J \in \mathbb{R}^{3}$ is the inertia matrix, $\theta \in \mathbb{R}^{3}$ is the attitude angle, $u_{\{r c s, \text { max }\}}$ denotes the moment of force produced by the RCS, and $u_{r c s}, u_{r w}$ are RCS and RW control inputs described as

$$
\begin{aligned}
& u_{r c s}:\{-1,0,1\} \in \mathbb{Z}^{3} \\
& u_{r w}: u_{\{r w, \min \}} \leq u_{r w} \leq u_{\{r w, \max \}} \in \mathbb{R}^{3}
\end{aligned}
$$

where $u_{\{r w, \min \}}$ and $u_{\{r w, \max \}}$ are the RW saturation torque levels.

An RCS can produce large torque inputs compared with RW, so it is useful for large angle rotations or high-speed switching maneuvers of heavy spacecraft, although it takes only integer values. On the other hand, RW inputs can take linear values but their maximum torque is limited. In view of this, the first objective of this paper is to investigate the feasibility of MPC attitude maneuver using RCS and RW singly and in combination and to derive a design algorithm which ensures closed-loop stability.

The stability of model predictive control with input constraints has been well discussed for continuous input systems ${ }^{(9)}$, and this is directly applicable to the case of RW control. However the stability of MPC by on-off inputs has not yet been discussed. Therefore, the second objective of this paper is to investigate the closed-loop stability of on-off MPC using RCS alone and RCS and RW in combination.

\section{Controller Design}

This section is organized as follows. We first formulate the optimization problem for onoff MPC. Then, we introduce the input-to-state stability definition, its Lyapunov-like condition 
and the stability condition of MPC following the results from an earlier study ${ }^{(13)}$, and we derive a theorem of stability for the proposed on-off MPC. Based on these results, we show a design algorithm for MPC attitude maneuvers.

\subsection{Model Predictive Control}

We consider the following linear discrete time-invariant systems transformed from eq. (1) by specifying the sampling time.

$$
x(k+1)=f(x(k), u(k))=F x(k)+E u(k)
$$

where $x(k) \in \mathbb{R}^{n}$ and $u(k) \in \mathbb{R}^{m}$ are respectively the state variable and the control input at the $k$ th-step, and $(F, E)$ is assumed to be controllable.

The outline of model predictive control is as follows. We first construct the predictive state trajectory from $x(k+1)$ to $x(k+N)$ from the $k$ th-step state $x(k)$, and these are expressed as $\mathbb{X}(k)=\left[x(k+1)^{T}, \ldots, x(k+N)^{T}\right]^{T}$ where $N$ is the prediction step number. In a similar way, the control sequence from step $k$ to step $k+N-1$ is described as $\mathbb{U}(k)=\left[u(k)^{T}, \ldots, u(k+N-1)^{T}\right]$. Then, a cost function is defined with $\mathbb{X}(k)$ and $\mathbb{U}(k)$ and is optimized at each step. From the obtained optimal control sequence $\mathbb{U}^{o}(k)=\left[u^{o}(k)^{T}, \ldots, u^{o}(k+N-1)^{T}\right]$, the first step input, i.e. $u(k)^{T}=u^{o}(k)^{T}$, is applied to the system. The procedure is repeated at every sampling time interval.

The predicted state trajectory $\mathbb{X}(k)$ in this case is constructed from eq. (4) using the state $x(k)$ as

$$
\mathbb{X}(k)=\mathbb{F} x(k)+\mathbb{E} \mathbb{U}(k)
$$

where each coefficient matrix is

$$
\mathbb{F}=\left[\begin{array}{c}
F^{1} \\
F^{2} \\
\vdots \\
F^{N}
\end{array}\right], \mathbb{E}=\left[\begin{array}{cccc}
F^{0} E & 0 & \ldots & 0 \\
F^{1} E & F^{0} E & 0 & \vdots \\
\vdots & \vdots & \ddots & 0 \\
F^{N-1} E & \ldots & F^{1} E & F^{0} E
\end{array}\right] .
$$

We then construct the following quadratic cost function with $\mathbb{X}(k)$ and $\mathbb{U}(k)$.

$$
\begin{aligned}
V_{N}(x(k), \mathbb{U}(k))= & \mathbb{X}(k)^{T} \mathbf{Q} \mathbb{X}(k)+\mathbb{U}(k)^{T} \mathbf{R} \mathbb{U}(k) \\
= & \sum_{i=k+1}^{k+N-1} x(i)^{T} Q x(i)+u(i-1)^{T} R u(i-1) \\
& +x(k+N)^{T} P x(k+N)
\end{aligned}
$$

where $\mathbf{Q}=\operatorname{diag}(Q, \ldots, P) \in \mathbb{R}^{n N \times n N}$ is symmetric positive definite and $\mathbf{R}=\operatorname{diag}(R, \ldots, 0) \in$ $\mathbb{R}^{m N \times m N}$ is symmetric positive semi-definite. The stage cost matrices $Q, R$ in eq. (7) are given as design parameters, which have similar roles as in the standard infinite horizon linear quadratic regulator, i.e. the larger $Q$ results in better control performance and the larger $R$ results in smaller control input. As for the terminal cost matrix $P$, it is obtained so as to guarantee the closed-loop stability ${ }^{(8),(9)}$. For this purpose, in Ref. (9), discrete algebraic Riccati equations (DARE) are introduced to make the finite horizon prediction equivalent into an infinite one. Later on, we discuss the stabilizing condition of $P$ for on-off MPC. Once we define the cost, the unconstrained MPC is achieved by solving the following problem at each step.

$$
\begin{array}{cl} 
& \min _{\mathbb{U}}\left\{V_{N}(x(k), \mathbb{U}(k))=V(\mathbb{X}(k), \mathbb{U}(k))=\mathbb{X}(k)^{T} \mathbf{Q} \mathbb{X}(k)+\mathbb{U}^{T}(k) \mathbf{R} \mathbb{U}(k)\right\} \\
\text { s.t. } & \\
x: & x(k+1)=F x(k)+E u(k) \\
\mathbb{U}: & u(k), u(k+1), \ldots, u(k+N-1)
\end{array}
$$

By taking account of the step reference input $x_{r}$ in problem (8) with the corresponding reference vector $\mathbb{X}_{r}=\left[x_{r}^{T}, \ldots, x_{r}^{T}\right]^{T}$ and the on-off property of control input $u_{i}=\{-1,0,1\}$, the 
optimization problem becomes an integer quadratic programming (IQP) problem as shown below.

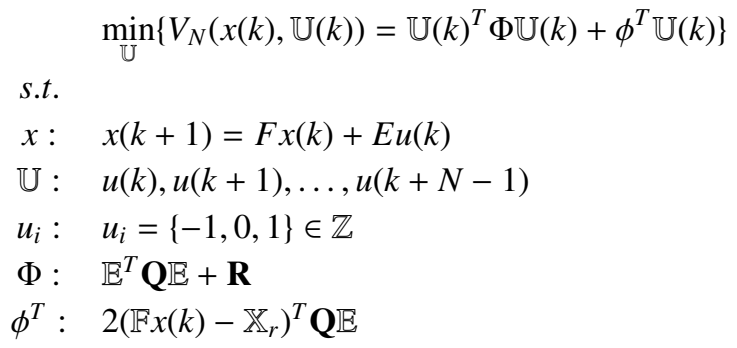

For spacecraft control by a combination of RW and RCS, new input variables and input saturation constraints for the RW are further added to eq. (9). In this case, the optimization problem becomes a mixed integer quadratic programming (MIQP) problem that includes integer and continuous optimization variables. MIQP has appeared mainly in MPCs for hybrid systems, which have logical binary variables and some controlled real variables. MIQP and IQP are numerically solvable by the branch and bound method ${ }^{(14)}$.

\subsection{Input-to-State Stability}

In this subsection, we provide input-to-state stability definitions and theorems in order to evaluate the on-off MPC closed-loop stability problem. The concept of input-to-state stability was introduced by Sontag ${ }^{(15)}$. Since the concept permits nonlinearlity of input variables in a Lyapunov-like stability framework, it is currently well-known as a useful tool for nonlinear control problems. Additionally, after Jiang and Wang ${ }^{(11)}$ applied it to discrete time systems, it has been widely used in nonlinear model predictive control and robust model predictive control for stability analysis ${ }^{(12),(16)}$.

Definition 1 (class- $\mathcal{K}$, class- $\mathcal{K}_{\infty}$ function)

A function $\gamma(s): \mathbb{R}_{\geq 0} \mapsto \mathbb{R}_{\geq 0}$ is a class- $\mathcal{K}$ function if it is continuous, strictly increasing and $\gamma(0)=0$. Additionally, if a class $-\mathcal{K}$ function satisfies $\gamma(s) \rightarrow \infty$ for the argument $s \rightarrow \infty$, then it is a class $-\mathcal{K}_{\infty}$ function.

\section{Definition 2 (class- $\mathcal{K} \mathcal{L}$ function)}

A function $\beta(s, t): \mathbb{R}_{\geq 0} \times \mathbb{R}_{\geq 0} \mapsto \mathbb{R}_{\geq 0}$ is a class $-\mathcal{K} \mathcal{L}$ function if $\beta(\cdot, t), \forall t \geq 0$ is a class $-\mathcal{K}$ function and $\beta(s, \cdot)$ monotonically decreases $\forall s \geq 0$ and $\beta(s, t) \rightarrow 0$ for $t \rightarrow \infty$.

\section{Definition 3 (Input-to-State Stable(ISS))}

The system eq. (4) is input-to-state stable if a class $-\mathcal{K} \mathcal{L}$ function $\beta$ and a class $-\mathcal{K}$ function $\gamma$ exist and the following property is satisfied for each step $k \in \mathbb{Z}_{+}$,

$$
|x(k, \xi, u)| \leq \beta(|\xi|, k)+\gamma(\|u\|)
$$

where $u \in l_{\infty}^{m}, x(0)=\xi \in \mathcal{D}^{n} \in \mathbb{R}^{n}$ (see Jiang and Wang ${ }^{(11)}$ ). Additionally, ISS becomes global asymptotic stability when $u=0$ and $\mathcal{D}^{n}=\mathbb{R}^{n}$ from the definition of $A S^{(17)}$.

\section{Definition 4 (ISS-Lyapunov function)}

The continuous value function $V: \mathbb{R}^{n} \mapsto \mathbb{R}$ is an ISS-Lyapunov function if there exist $\mathcal{K}_{\infty}$ functions $\alpha_{1}, \alpha_{2}, \alpha_{3}$ and a $\mathcal{K}$ function $\sigma$ such that

$$
\begin{aligned}
\alpha_{1}(|\xi|) & \leq V(\xi) \leq \alpha_{2}(|\xi|), \forall \xi \in \mathbb{R}^{n} \\
V(f(\xi, \mu))-V(\xi) & \leq-\alpha_{3}(|\xi|)+\sigma(|\mu|), \forall \xi \in \mathbb{R}^{n}, \mu \in \mathbb{R}^{m}
\end{aligned}
$$

where $\mu$ is the exogenous input variable applied to the system.

Based on the above definitions, the input-to-state stability condition is stated as follows.

\section{Theorem 1}

A system is ISS if and only if it admits an ISS-Lyapunov function. 


\section{Proof}

See the references ${ }^{(10),(11)}$.

Thus, ISS is the stability of the state when the system is under the influence of exogenous input variables. Therefore it gives the following internal stability condition when the system is free from the inputs, that is $\mu=0$ in eq. (11).

\section{Corollary 1}

A system is AS if and only if it admits the Lyapunov function which is obtained by excluding the $\sigma$ term from the ISS-Lyapunov function ${ }^{(17)}$.

\subsection{Stability of model predictive control}

The stability of MPC is investigated based on the Lyapunov second method or the monotonicity of cost functions, and it is known that the stability of the finite horizon optimization problem is ensured by inequality conditions with the stage cost and the terminal $\operatorname{cost}^{(13)}$. This paper uses these conditions to guarantee ISS by utilizing the fact that the cost function in eq. (7) is also available as a candidate for the ISS-Lyapunov function. Then, we show that the on-off MPC becomes at least input-to-state stable by proving that there exists a control law that satisfies Theorem 1 .

\section{Assumptions}

We now suppose the following assumptions.

[A1] $0 \in X_{f} \subset \Xi$ and $X_{f}$ is closed

[A2] $u_{N}\left(x_{N}\right) \in \Upsilon, \forall x_{N} \in X_{f}$

[A3] $f\left(x_{N}, u_{N}\right) \in X_{f}, \forall x_{N} \in X_{f}$

where $\Xi$ is the state constraint set, $\Upsilon$ is the input constraint set, and $X_{f}$ is the terminal constraint set. The assumptions [A1-A3] mean respectively that the state constraint $\Xi$ is satisfied in $X_{f}$, the control constraint $\Upsilon$ is satisfied in $X_{f}$, and $X_{f}$ is positively invariant under terminal control input $u_{N}$.

In our problem (9), $\Xi$ and $X_{f}$ are apparently unconstrained, i.e. they are equivalent to $\mathbb{R}^{n}$. The set $\Upsilon$ is integers $\{-1,0,1\}$ when RCS is used, and it is the region $u_{\{r w, \min \}} \leq u_{r w} \leq u_{\{r w, \max \}}$ when RW is used for control.

\section{Theorem 2}

Suppose the MPC cost function eq. (7) is a candidate ISS-Lyapunov function. Then, under assumptions [A1-A3], the origin of the closed-loop system is input-to-state stable if the following inequality is satisfied.

$$
\mathcal{F}^{*}\left(x_{N}\right)+\mathcal{L}\left(x_{N}, u_{N}\right) \leq-\alpha_{3}\left(\left|x_{N}\right|\right)+\sigma(|\mu|), \forall x_{N} \in X_{f}
$$

where the terminal cost difference $\mathcal{F}^{*}\left(x_{N}\right)$ and the stage cost $\mathcal{L}\left(x_{N}, u_{N}\right)$ are defined by

$$
\begin{aligned}
\mathcal{F}^{*}\left(x_{N}\right) & =\left|x_{N+1}\right|_{P}^{2}-\left|x_{N}\right|_{P}^{2} \\
\mathcal{L}\left(x_{N}, u_{N}\right) & =\left|x_{N}\right|_{Q}^{2}+\left|u_{N}\right|_{R}^{2} .
\end{aligned}
$$

and $x_{N}=x(k+N), u_{N}=u(k+N-1)$ are the terminal state and the terminal control input, respectively.

\section{Proof}

By the optimization problem eq. (8), we obtain the optimal trajectory $\mathbb{X}^{o}(k)$ and the optimal input sequence $\mathbb{U}^{o}(k)$ under assumptions [A1-A2].

$$
\begin{aligned}
& \mathbb{X}^{o}(k)=\left\{x^{o}(k+1)^{T}, x^{o}(k+2)^{T}, \ldots, x^{o}(k+N)^{T}\right\}^{T} \\
& \mathbb{U}^{o}(k)=\left\{u^{o}(k)^{T}, u^{o}(k+1)^{T}, \ldots, u^{o}(k+N-1)^{T}\right\}^{T}
\end{aligned}
$$

Therefore, one-step later, these are

$$
\begin{aligned}
& \mathbb{X}(k+1)=\left\{x^{o}(k+2)^{T}, \ldots, x^{o}(k+N)^{T}, x(k+N+1)^{T}\right\}^{T} \\
& \mathbb{U}(k+1)=\left\{u^{o}(k+1)^{T}, \ldots, u^{o}(k+N-1)^{T}, u(k+N)^{T}\right\}^{T}
\end{aligned}
$$


and the cost function becomes

$$
\begin{aligned}
V(\mathbb{X}(k+1), \mathbb{U}(k+1))= & V\left(\mathbb{X}^{o}(k), \mathbb{U}^{o}(k)\right) \\
& -\left|x^{o}(k+1)\right|_{Q}^{2}-\left|u^{o}(k)\right|_{R}^{2}-\left|x^{o}(k+N)\right|_{P}^{2} \\
& +\left|x^{o}(k+N)\right|_{Q}^{2}+\left|u^{o}(k+N-1)\right|_{R}^{2}+|x(k+N+1)|_{P}^{2}
\end{aligned}
$$

Now we evaluate the one-step difference $\Delta V$ defined by

$$
\Delta V=V(\mathbb{X}(k+1), \mathbb{U}(k+1))-V\left(\mathbb{X}^{o}(k), \mathbb{U}^{o}(k)\right) .
$$

From eq. (17) and using notations eq. (13), we obtain

$$
\Delta V+\mathcal{L}\left(x^{o}(k+1), u^{o}(k)\right)=\mathcal{F}^{*}\left(x^{o}(k+N)\right)+\mathcal{L}\left(x^{o}(k+N), u^{o}(k+N-1)\right) .
$$

If $\Delta V \leq-\alpha_{3}\left(\left|x_{N}\right|\right)+\sigma(|\mu|)$ is satisfied, $V_{N}(x(k), \mathbb{U}(k))$ is a ISS-Lyapunov function from Definition 4. By replacing $x^{o}(k+N)$ and $u^{o}(k+N-1)$ in eq. (18) with $x_{N}$ and $u_{N}$, we obtain the condition

$$
\Delta V \leq \mathcal{F}^{*}\left(x_{N}\right)+\mathcal{L}\left(x_{N}, u_{N}\right), \forall x_{N} \in X_{f},
$$

since $\mathcal{L}\left(x^{o}(k+1), u^{o}(k)\right)$ in eq. (18) is non-negative. Therefore, if eq. (12) is satisfied, $V_{N}(x(k)$, $\mathbb{U}(k))$ is an ISS-Lyapunov function. The condition eq. (12) implies [A3]; that is, they are in the common situation.

\section{Corollary 2}

Under assumptions[A1-A3], the origin of the closed-loop system with MPC is asymptotically stable if

$$
\mathcal{F}^{*}\left(x_{N}\right)+\mathcal{L}\left(x_{N}, u_{N}\right) \leq-\alpha_{3}\left(\left|x_{N}\right|\right), \forall x_{N} \in X_{f} .
$$

\section{Proof}

This is apparent from eq. (12) and Corollary 1.

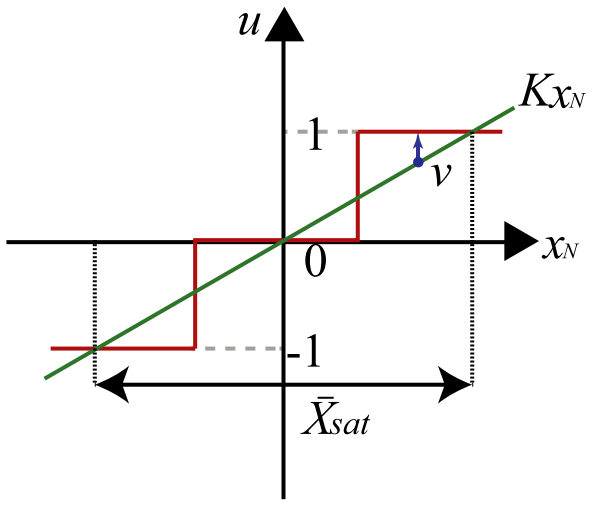

Fig. 1 Illustration of on-off property

In the case where control inputs are produced by RCS, we describe the control input $u_{N}\left(x_{N}\right)$ as the sum of a linear part $K x_{N}$ and a quantization error $v$ in the proper region shown schematically in Fig. 1. The value of $v$ is treated as the exogenous input hereafter. Then, the terminal cost matrix $P$ guaranteeing the stability is obtained by the following theorem.

\section{Theorem 3}

Assume that stage cost matrices are $Q, R>0$ and the terminal control law is $u_{N}\left(x_{N}\right)=K x_{N}+v$. Then, under assumptions [A1-A3], the origin of the closed-loop system is ISS if the terminal cost matrix $P$ satisfies

$$
x_{N}^{T}\left(S^{T} P S-P+Q+K^{T} R K\right) x_{N} \leq-\gamma x_{N}^{T} x_{N}+\sigma(|v|), \forall x_{N} \in \bar{X}_{s a t} \subseteq X_{f}
$$


where $K$ is a terminal controller gain that satisfies $S=F+E K$ being stable, $\bar{X}_{\text {sat }}=\{x \in$ $\left.\mathbb{R}^{n}|| K_{i} x \mid \leq 1 \forall i\right\}$ is the unsaturated state set for the control input $u_{N}\left(x_{N}\right)$ which consists of a linear controller gain $K$ and $v$, where $\gamma$ is a positive scalar value which gives the upper bound of $L M I$ and $\sigma(\cdot)$ is a class- $\mathcal{K}$ function of $v$.

\section{Proof}

We first suppose that the MPC cost function $V_{N}(x(k), \mathbb{U}(k))$ in eq. (7) is a candidate ISSLyapunov function. Then, Theorem 2 gives the cost function one-step difference as

$$
\mathcal{F}^{*}\left(x_{N}\right)+\mathcal{L}\left(x_{N}, u_{N}\right)=x_{N+1}^{T} P x_{N+1}-x_{N}^{T} P x_{N}+x_{N}^{T} Q x_{N}+u_{N}^{T} R u_{N} .
$$

The terminal control law $u_{N}\left(x_{N}\right)$ is supposed to consist of a stabilizing control input $K x_{N}$ and $v$, i.e.

$$
u_{N}\left(x_{N}\right)=K x_{N}+v
$$

and the $i$-th element of $v$ satisfies

$$
v_{i}=\left\{\left|v_{i}\right| \leq 1, \text { for }\left|K_{i} x_{N}\right| \leq 1\right\}
$$

for $i=1, \ldots, m$. Then, when the terminal control input $u_{N}\left(x_{N}\right)$ satisfies the input constraint $\left|K_{i} x_{N}\right| \leq 1, \forall i$, eq. (22) becomes as follows with $v$ from eq. (4) and eq. (23),

$$
e q .(22)=x_{N}^{T}\left(S^{T} P S-P+Q+K^{T} R K\right) x_{N}+2 v^{T} E^{T} P S x_{N}+v^{T}\left(E^{T} P E+R\right) v(25)
$$

where $S=(F+E K)$. When the terminal cost matrix $P$ satisfies

$$
S^{T} P S-P+Q+K^{T} R K \leq-\gamma I_{n},
$$

the following inequality is derived

$$
\begin{aligned}
e q .(22) & \leq-\gamma x_{N}^{T} x_{N}+2 v^{T} E^{T} P S x_{N}+v^{T}\left(E^{T} P E+R\right) v \\
& \leq-\gamma\left|x_{N}\right|^{2}+2\left\|E \left|\| | | P \| \left\|S\left|\|v\| x_{N}\right|+\left(\|E\|^{2}\|P\|+\|R\|\right)|v|^{2} .\right.\right.\right.
\end{aligned}
$$

Since an inequality $\left(\left|x_{N}\right| / z-z\|E|||P\||||S|\| v|)^{2} \geq 0\right.$ holds

$$
\left|x_{N}\right|^{2} / z^{2}+z^{2}\|E\|^{2}\|P\|^{2}\|S\|^{2}|v|^{2} \geq 2\left\|E \left|\left\||| P\left|\||S|\| v \| x_{N}\right|\right.\right.\right.
$$

Therefore,

$$
\begin{aligned}
e q .(27) & \leq-\gamma\left|x_{N}\right|^{2}+\left|x_{N}\right|^{2} / z^{2}+z^{2}\|E\|^{2}\|P\|^{2}\|S\|^{2}|v|^{2}+\left(\|E\|^{2}\|P\|+\|R\|\right)|v|^{2} \\
& \leq-\alpha_{3}\left(\left|x_{N}\right|\right)+\sigma(|v|)
\end{aligned}
$$

for an arbitrary scalar variable $\gamma>1 / z^{2}>0$, where $\alpha_{3}(r)=\left(\gamma-1 / z^{2}\right) r^{2}$ is a class $-\mathcal{K}_{\infty}$ function and $\sigma(r)=\left(z^{2}\|E\|^{2}\|P\|^{2}\|S\|^{2}+\|E\|^{2}\|P\|+\|R\|\right) r^{2}$ is a class- $\mathcal{K}$ function. The cost function $V_{N}$ is apparently an ISS-Lyapunov function, and therefore the closed-loop system is ISS from Theorem 1.

By comparing the cost function $V_{N}(x(k), \mathbb{U}(k))$ using $u_{N}\left(x_{N}\right)=K x_{N}+v$ with the optimal on-off MPC, the cost function $V_{N}\left(x(k), \mathbb{U}^{o}(k)\right)$ is in the relationship

$$
V_{N}(x(k), \mathbb{U}(k)) \geq V_{N}\left(x(k), \mathbb{U}^{o}(k)\right), \forall k \in \mathbb{Z}_{+}
$$

from the optimality. Therefore, the optimal on-off MPC also guarantees ISS.

\section{Corollary 3}

In the case of utilizing $R W$, the input $v$ is zero and so the $\sigma$ term in eq. (12) disappears. As a result, the closed-loop system is AS.

Therefore, we can conclude that if the terminal cost $x_{N}^{T} P x_{N}$ is constructed using the solution $P$ of the LMI eq. (26), the closed-loop system will be ISS for RCS control and AS for RW control. Even when MPC is performed with both RCS and RW in combination, it can be conjectured that RW will make the closed-loop system AS after the use of RCS causes states 
enter the ISS region. In the case where control is achieved by RW alone, it is well known that the solution $P$ of DARE can be used as a candidate of the terminal cost matrix to guarantee $\mathrm{AS}^{(8)}$. This can be proved using eq. (12).

\section{Corollary 4}

Assume that stage cost matrices are $Q, R>0$. Then, under the assumptions [A1-A3], the origin of the closed-loop system is asymptotically stable if the terminal cost matrix $P$ satisfies the discrete algebraic Riccati equation (DARE).

$$
F^{T} P F-P+Q-F^{T} P E\left(E^{T} P E+R\right)^{-1} E^{T} P F=0
$$

\section{Proof}

Let the terminal control law $u_{N}\left(x_{N}\right)=K x_{N}$ where $K=\left(E^{T} P E+R\right)^{-1} E^{T} P F$. Substitute it into eq. (21), we obtain

$$
x_{N}^{T}\left(F^{T} P F-P+Q-F^{T} P E\left(E^{T} P E+R\right)^{-1} E^{T} P F\right) x_{N}=0, \forall x_{N} \in \bar{X}_{s a t} \subseteq X_{f} .
$$

where $\bar{X}_{\text {sat }}=\left\{x \in \mathbb{R}^{n} \mid u_{\text {min }} \leq u_{N}(x) \leq u_{\text {max }}\right\}$ is the unsaturated state set for control constraints.

As described in the proof, the solution $P$ of DARE (31) is included in the solution set of the LMI (26). This fact implies that the LMI condition (26) is superior and gives greater flexibility in designing the terminal cost for MPC.

\subsection{Algorithm of the proposed MPC}

In summary, the MPC which stabilizes the closed-loop system is designed by the following procedure.

\section{Off-line Design:}

Given arbitrary stage cost matrices $Q, R>0$, the controller gain $K$ is designed so as to satisfy $F+E K$ being stable. It is preferable to make the gain $K$ as small as possible in order to enlarge the unsaturated set $\bar{X}_{\text {sat }}$ and the stability region. Then the LMI eq. (26) is solved to obtain terminal cost $P$. At this stage, it must be confirmed whether the unsaturated set $\bar{X}_{\text {sat }}$ satisfies the practical region of control by checking following inequalities for satisfying the input constraint set $\Upsilon$ at the terminal state. That is,

$$
\left|K x_{0}\right| \leq \| K|| x_{0} \mid \leq \max \left(\left|u_{\min }\right|,\left|u_{\max }\right|\right)
$$

when RW is used, and

$$
\left|K x_{0}\right| \leq \| K||\left|x_{0}\right| \leq 1
$$

when RCS or RCS plus RW are used. If yes, then construct the stage cost matrices $\mathbf{Q}, \mathbf{R}$ and proceed to the following on-line optimization Step 1 below according to eq. (7). Otherwise, redesign the abstract controller gain $K$ by decreasing it as long as $F+E K$ is stable.

\section{On-line Optimization:}

Step 1: Obtain the present state variable $x(k)$ and solve the optimization problem (9).

Step 2: Apply the first input of the optimized control sequence $\mathbb{U}^{o}$ as $u(k)$ to the system. Step 3: Return to Step1.

\section{Numerical Example}

Using the parameters shown in Table 1, we carried out numerical simulations for the following three cases to confirm the effectiveness of the proposed MPC design algorithm. Calculations were performed using YALMIP ${ }^{(18)}$ and the MPT toolbox ${ }^{(19)}$.

Case 1: We first compare the performance of an MPC attitude maneuvers with RW obtained using the terminal cost matrix $P$ determined by LMI in eq. (26) and DARE in eq. (31). Fig. 2 shows the roll attitude responses to a 1-degree step command and the control inputs. 
Table 1 Parameters of numerical study

\begin{tabular}{|c|c|c|c|c|}
\hline Parameter & \multicolumn{4}{|c|}{ Parameter value } \\
\hline Inertia $J\left[\mathrm{kgm}^{2}\right]$ & $\begin{array}{c}6.0701 \\
-0.0163 \\
-0.0974\end{array}$ & $\begin{array}{c}-0.0163 \\
6.9443 \\
0.0119\end{array}$ & $\begin{array}{c}-0.0974 \\
0.0119 \\
8.9646\end{array}$ & $\times 10^{4}$ \\
\hline Prediction steps $N$ & \multicolumn{4}{|c|}{10} \\
\hline Sampling time [sec] & \multicolumn{4}{|c|}{1} \\
\hline RCS output $[\mathrm{Nm}]$ & \multicolumn{4}{|c|}{100} \\
\hline RW output [Nm] & \multicolumn{4}{|c|}{0.04} \\
\hline Stage cost matrix & \multicolumn{4}{|c|}{$Q=10 \mathrm{E}-1 * I_{n}, R=10 \mathrm{E}-3 * I_{m}$} \\
\hline Case1: & \multicolumn{4}{|c|}{ Comparison of terminal cost matrix $(3000[\mathrm{sec}])$} \\
\hline Initial angle[deg] & \multicolumn{4}{|c|}{$[0,0,0]$} \\
\hline Target angle[deg] & \multicolumn{4}{|c|}{$[1,0,0]$} \\
\hline Case2: & \multicolumn{4}{|c|}{ Attitude step maneuver(300[sec]) } \\
\hline Initial angle[deg] & \multicolumn{4}{|c|}{$[0,0,0]$} \\
\hline Target angle[deg] & \multicolumn{4}{|c|}{$[1,0,0]$} \\
\hline Case 3: & \multicolumn{4}{|c|}{ Switching maneuver(300[sec]) } \\
\hline Initial angle[deg] & \multicolumn{4}{|c|}{$[0,0,0]$} \\
\hline Target angle[deg] & \multicolumn{4}{|c|}{$[10,0,0](100[\mathrm{sec}]) \rightarrow[0,0,0](200[\mathrm{sec}]) \rightarrow[10,0,0](300[\mathrm{sec}])$} \\
\hline
\end{tabular}
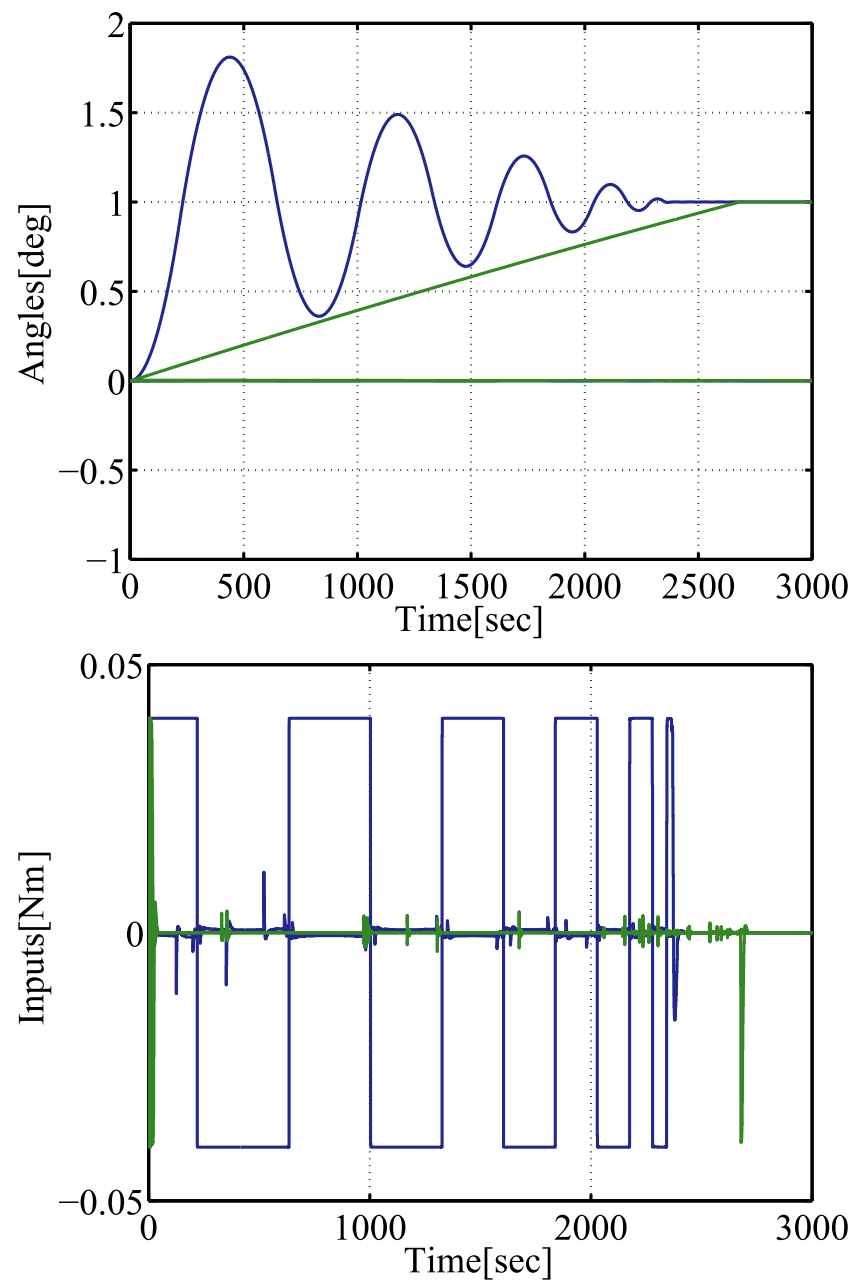

Fig. 2 Attitude responses(top) and control inputs(bottom): DARE(- - LMI(-)

Other attitude commands are set to zero. We first find that both MPCs converge to the commanded angle and are therefore asymptotically stable. It is also found that dynamic coupling with other axes is negligible. However the response of MPC based on DARE is oscillatory and input saturation occurs repeatedly, although the resultant settling time is similar to that obtained using LMI, and a larger control input is required as a result. This is caused by the fact that the unique solution $P$ of DARE is not sufficiently large and so the terminal cost is 


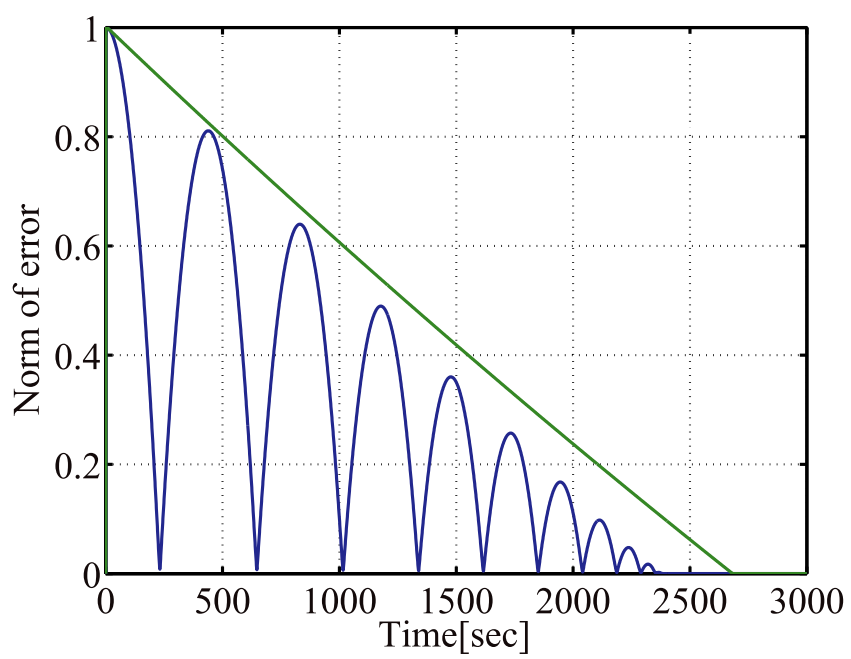

Fig. 3 Norms of attitude error: DARE $(-), \operatorname{LMI}(-)$
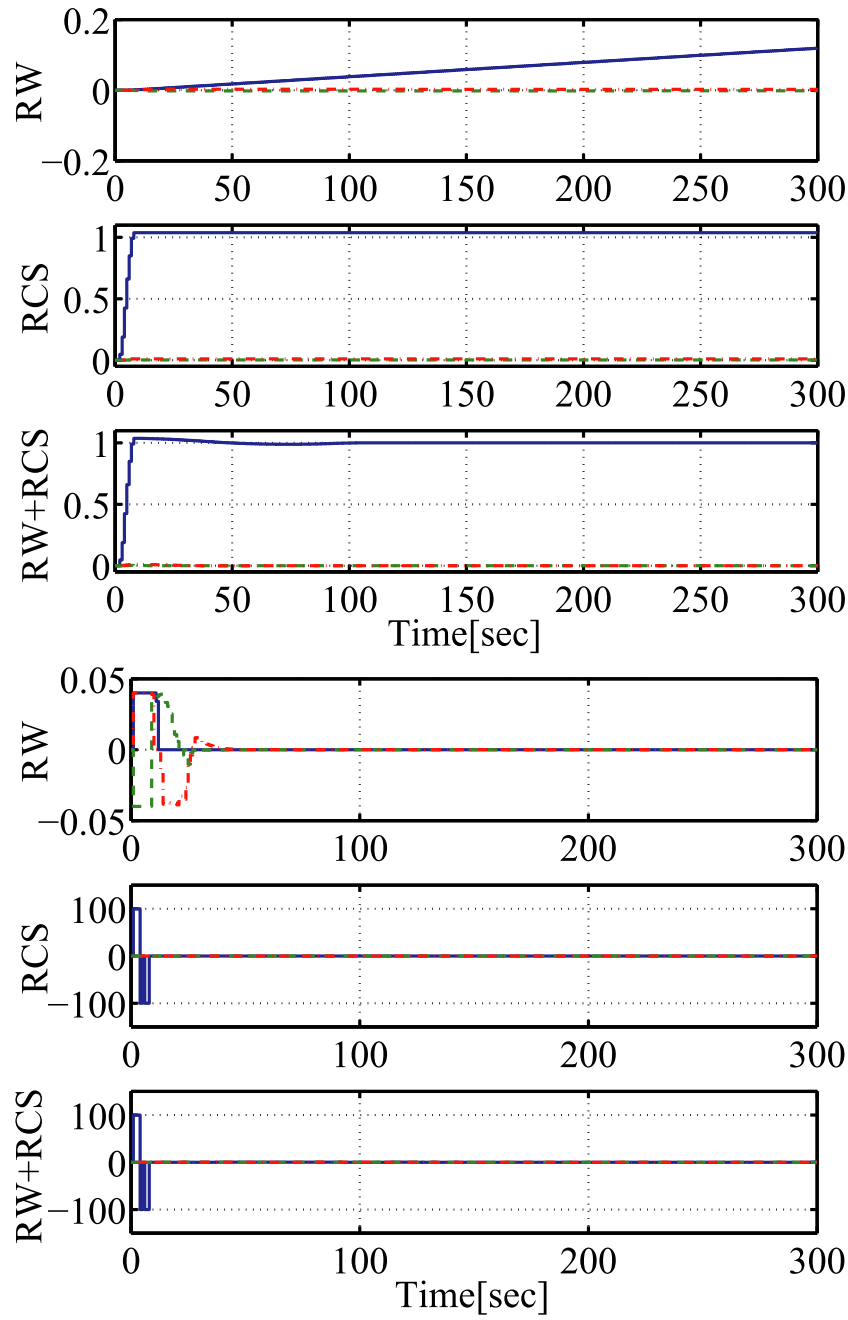

Fig. 4 Attitude responses(top) and control inputs(bottom): $\mathrm{x}(-), \mathrm{y}(---), \mathrm{z}(\cdot-\cdot-\cdot)$

small, while $P$ obtained by LMI (26) can be made larger by setting a small terminal feedback gain $K$. The result demonstrates that the LMI-based design has greater flexibility than the DARE-based one. The norms of attitude errors are shown in Fig. 3. Therefore, in the two simulation cases below, only the terminal cost obtained by LMI eq. (26) is considered.

Case 2: We compare the control performance of a 1-degree attitude step maneuver given by (a) using RCS alone and RCS and RW in combination in which the optimizations are 


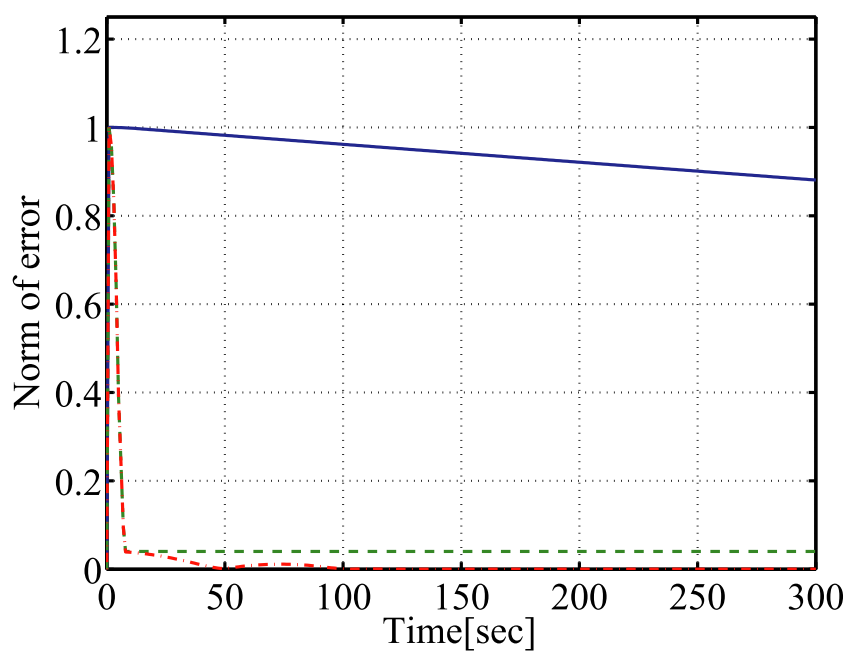

Fig. 5 Norms of attitude error: RW(- - , RCS(-- -), RW+RCS(----·)
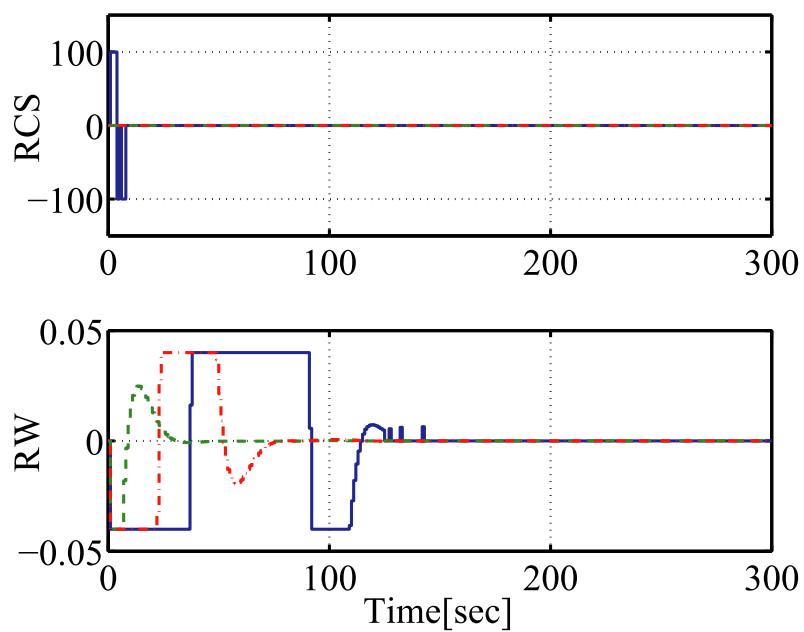

Fig. 6 Control inputs of RCS(top) and RW(bottom): x(-), y(-- -), z(----·)

based on MIQP, and (b) using only RW, which has already been shown in Fig. 2. From the time histories of attitude response and total control inputs shown in Fig. 4, we observe that the maneuver using RCS alone is only ISS and an attitude bias of about 0.05 [deg] remains, and that the responses of the maneuver using RW alone is not desirable compared with the RCS results although it is AS. Only the combined use of RCS and RW gives rapid response and asymptotic stability. The control performance is also confirmed from the norms of the attitude error shown in Fig. 5. Fig. 6 shows the inputs of RCS and RW separately in the case of an attitude maneuver using RCS and RW in combination. The RCS operates only during the initial 10 [sec] period; the state variables enter the ISS stable region and after that the RW works to asymptotically stabilize the system. Finally, all the states are driven until they coincide with the command inputs. In Fig. 7, we also show the on-line optimization time at each step when CPLEX ${ }^{(20)}$ is used in Matlab environment. It is obvious that the computation time in this case is shorter that the sampling period 1 [sec].

Case 3:Finally, we apply all types of MPC to the switching maneuver problem which changes roll angle repeatedly from an initial $0[\mathrm{deg}]$ at $0[\mathrm{sec}]$ to $10[\mathrm{deg}]$ and then back, each change occuring at $100[\mathrm{sec}]$ intervals, while maintaining pitch and yaw angles at 0 [deg]. It is apparent that the RW MPC cannot follow the repeating commands because of its slow response, and that the RCS MPC follows the commands well but with a bias (Fig. 8). The norms of error angles are shown in Fig. 9. The only entirely successful case is the combined use of RCS and RW. The RCS and RW inputs are shown separately in Fig. 10. 


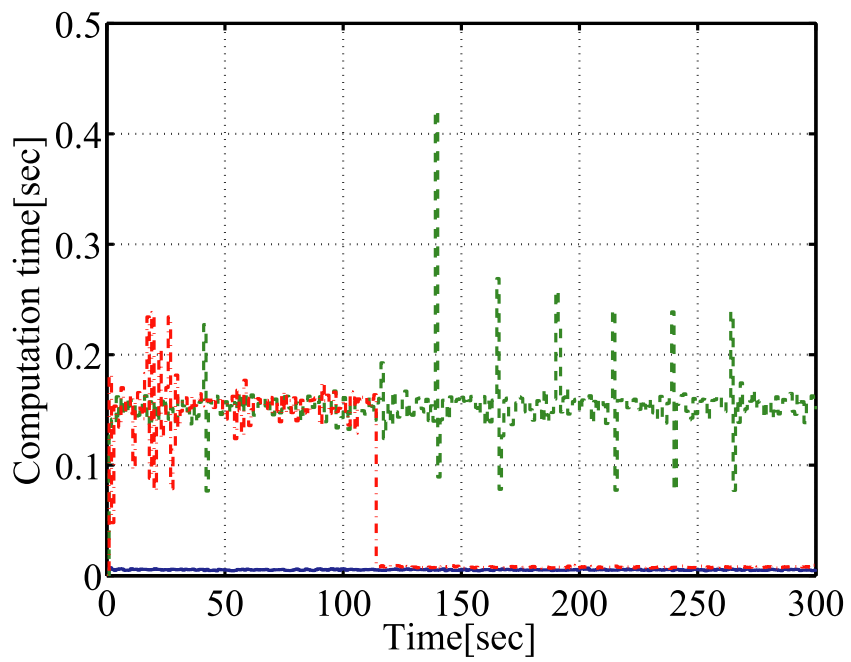

Fig. 7 Computation time: RW(- - , RCS(---), RW+RCS(----)
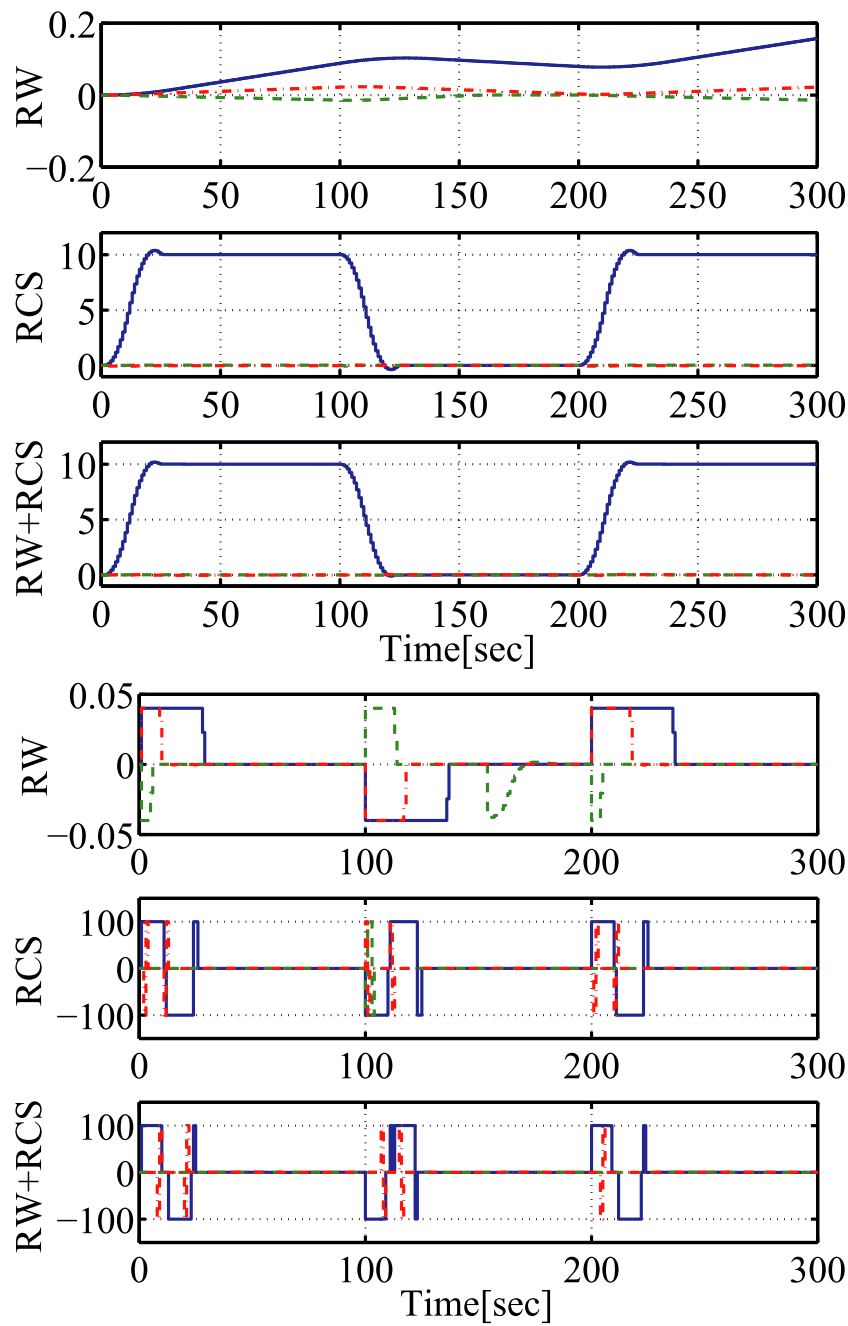

Fig. 8 Attitude responses(top) and control inputs(bottom): $\mathrm{x}(-), \mathrm{y}(---), \mathrm{z}(\cdot-\cdot-\cdot)$

From above results, we conclude that the stability conditions on ISS of RCS MPC and AS of RW and RCS plus RW are numerically confirmed, and that the feasibility of all types of MPC is successfully demonstrated. From a practical viewpoint, the combined use of RCS and RW is the most effective in terms of control performance and stability. 


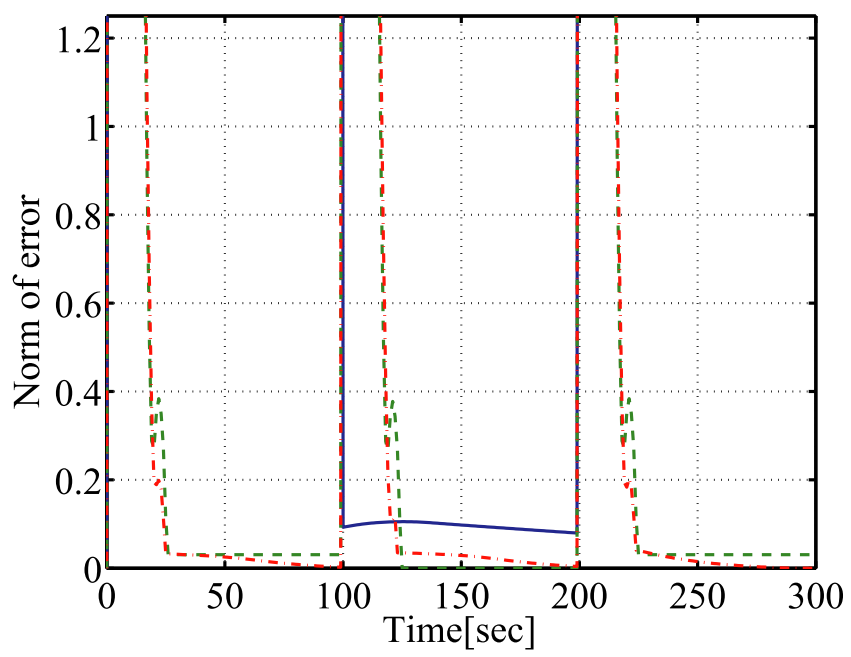

Fig. 9 Norms of attitude error: RW(- - , RCS(- - -), RW+RCS(----)
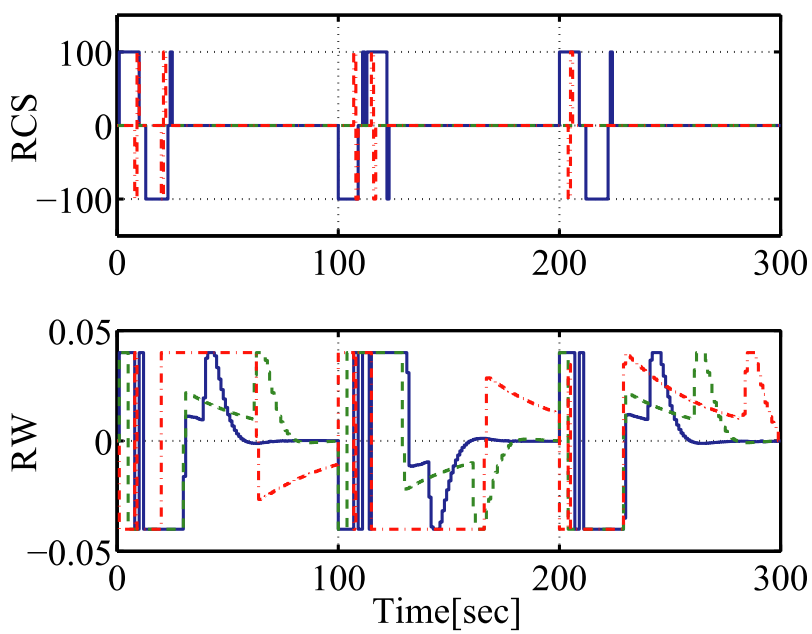

Fig. 10 Control inputs of RCS(top) and RW(bottom): x(-), y(- - -), z(----·)

\section{Conclusion}

This paper has investigated the MPC problem for spacecraft attitude maneuvers using RCS and/or RW. For this purpose, we have derived a stability condition described by LMI to prove that the closed-loop system becomes input-to-state stable for the RCS control case and becomes asymptotically stable for RW control. We further provided numerical study results to show the ability of the proposed MPC control algorithm using MIQP. Our remaining work is to demonstrate the feasibility through hardware experiments. For large attitude maneuvering, the control moment gyro (CMG) is also a promising actuator although it has nonlinear dynamics and so-called singular problems. Investigation of MPC using CMG is also one of our future research topics.

\section{References}

( 1 ) Attitude control research committee, ed., Spacecraft dynamics and control handbook (in Japanese). Baifukan, 2007.

( 2 ) Somov, Y., Butyrin, S., and Somov, S., "Guidance and robust gyromoment precise attitude control of agile observation spacecraft," in Proceedings of the 17th IFAC World Congress, pp. 3422-3427, 2008.

( 3 ) Tanaka, T., Kida, T., Nagashio, T., Ohtani, T., Yamaguchi, I., Kasai, T., Hamada, Y., Sakai, S., Bando, N., "A preliminary study on precise attitude switching maneuver of 
flexible spacecraft using cmg," Journal of Space Engineering, Vol. 2, No. 1, pp. 12-24, 2009.

( 4 ) "Studies in astronautics," in Optimal Spacecraft Rotational Maneuvers (Junkins, J. L. and Turner, J. D., eds.), Vol. 3 of Studies in Astronautics, Elsevier, 1986.

( 5 ) Hegrenæs, Ø., Gravdahl, J. T., and Tøndel, P., "Spacecraft attitude control using explicit model predictive control," Automatica, Vol. 41, No. 12, pp. 2107-2114, 2005.

( 6 ) Wen, J. T., Seereeram, S., and Bayard, D. S., "Nonlinear predictive control applied to spacecraft attitude control," in American Control Conference, Vol. 3, pp. 1899-1903, 1997.

( 7 ) Huang, R., Hwang, I., and Corless, M., "A new nonlinear model predictive control algorithm using differential transformation with application to interplanetary low-thrust trajectory tracking," in American Control Conference, pp. 4868-4873, 2009.

( 8 ) Maciejowski, J. M., Predictive Control with Constraints. Prentice Hall, first ed., 2000.

( 9 ) Rawlings, J. B. and Muske, K. R., "The stability of constrained receding horizon control," IEEE Transactions on Automatic Control, Vol. 38, No. 10, pp. 1512-1516, 1993.

(10) Sontag, E. D., "Input to state stability: Basic concepts and results," in Nonlinear and Optimal Control Theory (Nistri, P. and Stefani, G., eds.), Vol. 1932 of Lecture Notes in Mathematics, pp. 163-220, Springer Berlin Heidelberg, 2008.

(11) Jiang, Z.-P. and Wang, Y., "Input-to-state stability for discrete-time nonlinear systems," Automatica, Vol. 37, pp. 857-869, 2001.

(12) Magni, L., Raimondo, D. M., and Scattolini, R., "Regional input-to-state stability for nonlinear model predictive control," IEEE Transactions on Automatic Control, Vol. 51, No. 9, pp. 1548-1553, 2006.

(13) Mayne, D. Q., Rawlings, J. B., Rao, C. V., Scokaert, P. O. M., "Constrained model predictive control: stability and optimality," Automatica, Vol. 36, pp. 789-814, 2000.

(14) Bemporad, A. and Morari, M., "Control of systems integrating logic, dynamics and constraints," Automatica, Vol. 35, pp. 407-427, 1999.

(15) Sontag, E. D., "Smooth stabilization implies coprime factorization," IEEE Transactions on Automatic Control, Vol. 34, No. 4, pp. 435-443, 1989.

(16) Limon, D., Alamo, T., Raimondo, D. M., Peña, D. M., Bravo, J. M., Ferramosca, A., and Camacho, E. F., "Input-to-state stability: A unifying framework for robust model predictive control," in Nonlinear Model Predictive Control (Magni, L., Raimondo, D. M., and Allgöwer, F., eds.), Vol. 384 of Lecture Notes in Control and Information Sciences, pp. 1-26, Springer Berlin Heidelberg, 2009.

(17) Khalil, H. K., Nonlinear Systems Third Edition. Prentice Hall, third ed., 2002.

(18) Löfberg, J., "Yalmip : A toolbox for modeling and optimization in MATLAB," in Proceedings of the CACSD Conference, (Taipei, Taiwan), pp. 284-289, 2004.

(19) Kvasnica, M., Grieder, P., and Baotić, M., "Multi-Parametric Toolbox (MPT)." http: //control. ee. ethz.ch/ mpt/, 2004.

(20) IBM Corp., "IBM ILOG CPLEX optimizer." http://www- 01 .ibm.com/software/ commerce/optimization/cplex-optimizer/", 2012. 